Journal of Applied AnALysis

Vol. 14 , No. 1 (2008), pp. 73-88

\title{
GENERAL MIXED VECTOR F-IMPLICIT COMPLEMENTARITY PROBLEMS IN BANACH SPACES
}

\author{
K.-Q. WU and N.-J. HUANG \\ Received May 30, 2007 and, in revised form, November 29, 2007
}

\begin{abstract}
In this paper, we introduce a new class of general mixed vector $F$-implicit complementarity problems and general mixed vector $F$-implicit variational inequality problems, and study the equivalence between of them under certain assumptions in Banach spaces. We also derive some new existence theorems of solutions for the general mixed vector $F$-implicit complementarity problems and the general mixed vector $F$-implicit variational inequality problems by using the FKKM theorem under some suitable assumptions without monotonicity. Moreover, we establish sufficient conditions for the upper semicontinuity and lower semicontinuity of the solution mapping of the general mixed vector $F$ implicit variational inequality problems.
\end{abstract}

2000 Mathematics Subject Classification. Primary: 90C33, 49J40.

Key words and phrases. Mixed vector $F$-implicit complementarity problem, mixed vector $F$-implicit variational inequality, KKM-mapping, positively homogeneous mapping, ordered Banach space, upper semicontinuity, lower semicontinuity.

This work was supported by the National Natural Science Foundation of China (10671135), the Specialized Research Fund for the Doctoral Program of Higher Education (20060610005) and the Open Fund (PLN0703) of State Key Laboratory of Oil and Gas Reservoir Geology and Exploitation (Southwest Petroleum University).

ISSN 1425-6908（C) Heldermann Verlag. 


\section{INTRODUCTION AND MAIN RESULTS}

The complementarity theory is a new domain of applied mathematics and it has many applications in optimization, economics, finance, engineering, mechanics and game theory (see, for example, $[6,7,15,16]$ and the references therein). It is well known that some complementarity problems are equivalent to the related variational inequalities under certain conditions.

Vector variational inequality was first introduced and studied by Giannessi [11] in the setting of the finite-dimensional Euclidean spaces. Later on, Chen and Yang [3] considered general vector variational inequalities and vector complementary problems in infinite dimensional spaces. Since then, existence results of solutions for several kinds of vector variational inequalities and vector complementarity problems have been derived. At the same time, vector variational inequalities and vector complementarity problems have found many of its applications in vector optimization, set-valued optimization, approximate analysis of vector optimization problems and vector network equilibrium problems. For details, we refer to $[4,12,14]$ and the references therein.

In 2001, Yin, $\mathrm{Xu}$ and Zhang [20] introduced a class of $F$-complementarity problems (F-CP) for finding $x \in K$ such that

$$
\langle T x, x\rangle+F(x)=0 \quad \text { and } \quad\langle T x, y\rangle+F(y) \geq 0, \quad \forall y \in K,
$$

where $K$ is a nonempty closed convex cone of a real Banach space $X$, $T: K \rightarrow X^{*}$, the dual space, is a mapping and $F: K \rightarrow(-\infty,+\infty)$ is a function, and proved that it is equivalent to the following generalized variational inequality problems: find $x \in K$ such that

$$
\langle T x, y-x\rangle+F(y)-F(x) \geq 0, \quad \forall y \in K,
$$

where $K$ is a nonempty closed convex cone and $F$ is a positively homogeneous and convex function. They also proved the existence of solutions for (F-CP) under some assumptions with $F$-pseudomonotonicity.

Recently, by using the combination of demicontinuity and pseudomonotonicity, Fang and Huang [9] introduced and studied a new class of vector $F$-complementarity problems with demipseudomonotone mappings in Banach spaces. They also presented the solvability of this class of vector $F$ complementarity problems with demipseudomonotone mappings and finitedimensional continuous mappings in reflexive Banach spaces. Huang and Li [13] introduced and studied a class of scalar $F$-implicit complementarity problems and another class of $F$-implicit variational inequality problems in Banach spaces. In 2006, Li and Huang [18] generalized the result from the scalar case in [13] to the vector case. They proved the equivalence between 
the vector $F$-implicit complementarity problem and vector $F$-implicit variational inequality problem and obtained some new existence theorems for solutions for vector $F$-implicit complementarity problems.

Very recently, Lee, Khan, and Salahuddin [17] introduced a new class of generalized vector $F$-implicit complementarity problems and a corresponding new class of generalized vector $F$-implicit variational inequality problems in Banach spaces, which generalized some results of $[13,18]$ to a more generalized vector case. The equivalence between the generalized $F$-implicit complementarity problem and generalized $F$-implicit variational inequality problem was presented, and some new existence theorems of solutions for generalized $F$-implicit complementarity problems and generalized $F$-implicit variational inequality problems were also proved in [17].

Inspired and motivated by the above works, in this paper, we introduce a new class of general mixed vector $F$-implicit complementarity problems and general mixed vector $F$-implicit variational inequality problems, and study the equivalence between of them under certain assumptions in Banach spaces. We also derive some new existence theorems of solutions for the general mixed vector $F$-implicit complementarity problems and the general mixed vector $F$-implicit variational inequality problems by using the FKKM theorem under some suitable assumptions without monotonicity. Moreover, we establish sufficient conditions for the upper semicontinuity and lower semicontinuity of the solution mapping of the general mixed vector $F$-implicit variational inequality problems. The results presented in this paper extend and improve the corresponding results of works $[9,13,17,18]$.

\section{Preliminaries}

Let $Y$ be a real Banach space. Let $P \subset Y$ be a nonempty closed convex and pointed cone with the apex at the origin, that is, $P$ is a closed set with the following conditions:

(i) $\lambda P \subset P, \quad \forall \lambda>0$;

(ii) $P+P \subset P$;

(iii) $P \cap(-P)=\{0\}$.

An ordered Banach space $(Y, P)$ is a real Banach space $\mathrm{Y}$ with an ordering defined by a closed cone $P \subset Y$ with an apex at the origin as follows: for any $x, y \in Y$,

$x \geq y \Longleftrightarrow x-y \in P$

$x \nsupseteq y \Longleftrightarrow x-y \notin P$.

Definition 2.1. Let $X$ and $Y$ be vector spaces and $K$ a cone of $X$. A mapping $F: K \rightarrow Y$ is said to be positively homogeneous if $F(\alpha x)=\alpha F(x)$ for all $x \in K$ and $\alpha \geq 0$. 
Definition 2.2. A mapping $G: M \subset X \rightarrow 2^{X}$ is said to be a KKM mapping if, for any finite set $A \subset M$, co $A \subset \bigcup_{x \in A} G(x)$, where $2^{X}$ denotes the family of all the nonempty subsets of $X$ and $\operatorname{co} A$ is the convex hull of $A$.

Lemma 2.1 (FKKM theorem [8]). Let $M$ be a nonempty subset of a Hausdorff topological vector space $X$ and $G: M \subset X \rightarrow 2^{X}$ be a KKM mapping. If $G(x)$ is closed in $X$ for every $x \in M$ and compact for some $x \in M$, then $\bigcap_{x \in M} G(x) \neq \emptyset$.

Lemma $2.2([18])$. Let $(Y, P)$ be an ordered Banach space induced by a pointed closed convex cone $P$. Then $x \geq 0$ and $y \geq 0$ imply that $x+y \geq 0$, for all $x, y \in Y$.

A set-valued mapping $W: E \subset X \rightarrow Y$ is upper semicontinuous at $x_{0} \in$ $E$ if for every open set $V$ containing $W\left(x_{0}\right)$ there exists an open set $U$ containing $x_{0}$ such that $W(U) \subset V$. W is lower semicontinuous at $x_{0} \in E$ if every open set $V$ intersecting $W\left(x_{0}\right)$ there exists an open set $U$ containing $x_{0}$ such that $W(x) \cap V \neq \emptyset$ for every $x \in U$. $W$ is upper semicontinuous (lower semicontinuous) on $E$ if it is upper semicontinuous (lower semicontinuous) at every point of $E$. $W$ is continuous on $E$ if it is both upper semicontinuous and lower semicontinuous on $E$.

Lemma 2.3 ([10]). Let $W: X \rightarrow Y$ be a set-valued mapping.

(i) If for any $x \in X, W(x)$ is compact, then $W$ is upper semicontinuous at $x_{0}$ if and only if for any net $\left\{x_{\alpha}\right\} \subset X$ such that $x_{\alpha} \rightarrow x_{0}$ and for every $y_{\alpha} \in W\left(x_{\alpha}\right)$, there exists $y_{0} \in W\left(x_{0}\right)$ and a subnet $\left\{y_{\alpha_{i}}\right\}$ of $\left\{y_{\alpha}\right\}$ such that $y_{\alpha_{i}} \rightarrow y_{0}$.

(ii) $W$ is lower semicontinuous at $x_{0} \in X$ if and only if for any net $\left\{x_{\alpha}\right\} \subset$ $X$ with $x_{\alpha} \rightarrow x_{0}$, and for any $y_{0} \in W\left(x_{0}\right)$, there exists a net $\left\{y_{\alpha}\right\}$ such that $y_{\alpha} \in W\left(x_{\alpha}\right)$ and $y_{\alpha} \rightarrow y_{0}$.

\section{EXISTENCE RESUlTS}

In this section, unless otherwise specified, we suppose that $K$ is a nonempty closed convex cone of a real Banach space $X$ and $(Y, \leq)$ is an ordered Banach space induced by a pointed closed convex cone $P$. We

will discuss the following general mixed vector $F$-implicit complementarity problem (GMVF-ICP): find $x \in K$ such that

$$
Q(x, h(x))+F(h(x))=0
$$

and

$$
Q(x, g(y))+F(g(y)) \geq 0, \quad \forall y \in K
$$




\section{Some Special Cases}

(1) If $h(x)=g(x)$ for all $x \in K$, the (GMVF-ICP) collapses to the general vector $F$-complementarity problem $(\mathrm{GVF}-\mathrm{CP})$ of finding $x \in K$ such that

$$
Q(x, g(x))+F(g(x))=0
$$

and

$$
Q(x, g(y))+F(g(y)) \geq 0, \quad \forall y \in K
$$

(2) Let $A, T: K \rightarrow L(X, Y), g: K \rightarrow K, F: K \rightarrow Y$ and $N: L(X, Y) \times$ $L(X, Y) \rightarrow L(X, Y)$ be mappings, where $L(X, Y)$ is the space of all continuous linear operators from $X$ to $Y$. If we set $Q(x, y)=$ $\langle N(A x, T x), y\rangle$, the (GMVF-ICP) collapses to the generalized vector $F$-implicit complementarity problem (GVF-CP) of finding $x \in K$ such that

$$
\langle N(A x, T x), g(x)\rangle+F(g(x))=0
$$

and

$$
\langle N(A x, T x), g(y)\rangle+F(g(y)) \geq 0, \quad \forall y \in K
$$

which was considered and studied by Lee, Khan, and Salahuddin [17].

(3) Let $g$ is an identity mapping on $K$, the (GMVF-ICP) collapses to the vector $F$-complementarity problem of finding $x \in K$ such that

$$
Q(x, h(x))+F(h(x))=0
$$

and

$$
Q(x, y))+F(y) \geq 0, \quad \forall y \in K
$$

(4) If $F=0$, then we obtain the following vector complementarity problem

$$
Q(x, h(x))=0
$$

and

$$
Q(x, g(y))) \geq 0, \quad \forall y \in K .
$$

Summing up the above arguments, it shows that, for a suitable choice of the mappings $Q, g, h, F$ and the spaces $X$ and $Y$, we can obtain a number of known and new classes of complementarity problems, which include many complementarity problems studied in $[2]-[6],[9,11,12,13],[15]-[20]$ as special cases. It is worthy noting that problems (3.2)-(3.4) are all new.

We also introduce the following general mixed vector $F$-implicit variational inequality problem (GMVF-IVIP): find $x \in K$ such that

$$
Q(x, g(y))-Q(x, h(x))+F(g(y))-F(h(x)) \geq 0, \quad \forall y \in K .
$$

We first establish the equivalence between (GMVF-ICP) and (GMVFIVIP). 
Theorem 3.1. The following conclusions hold:

(i) If $x$ solves (GMVF-ICP), then $x$ solves (GMVF-IVIP).

(ii) Assume that $F: K \rightarrow Y$ is a positively homogeneous mapping, $Q: K \times$ $K \rightarrow Y$ is positively homogeneous in the second argument, and $g: K \rightarrow$ $K$ is surjective. If $x$ solves (GMVF-IVIP), then $x$ solves (GMVF$I C P)$.

Proof. (i) Suppose that $x$ solves (GMVF-ICP). Then it is easy to see that $x$ solves (GMVF-IVIP).

(ii) Let $x \in K$ be a solution of (GMVF-IVIP). Then

$$
Q(x, g(y))-Q(x, h(x))+F(g(y))-F(h(x)) \geq 0, \quad \forall y \in K .
$$

Since $F: K \rightarrow Y$ is a positively homogeneous mapping and $K$ is a convex cone, letting $g(y)=2 h(x)$ and $g(y)=h(x) / 2$ in (3.5), we have

$$
Q(x, h(x))+F(h(x)) \geq 0
$$

and

$$
Q(x, h(x))+F(h(x)) \leq 0 .
$$

Since $\mathrm{P}$ is a pointed cone, then

$$
Q(x, h(x))+F(h(x))=0 .
$$

Moreover, we obtain

$$
\begin{aligned}
Q(x, g(y))+F(g(y))= & Q(x, g(y))-Q(x, h(x))+F(g(y))-F(h(x)) \\
& +Q(x, h(x))+F(h(x)) \\
= & Q(x, g(y))-Q(x, h(x))+F(g(y))-F(h(x)) \\
\geq & 0
\end{aligned}
$$

for all $y \in K$. Hence $x$ solves (GMVF-ICP). The proof is completed.

Now we consider the existence of solutions to (GMVF-IVIP) and the properties of the solution sets.

Theorem 3.2. Let $K$ be nonempty closed and convex subset of $X$. Assume that

(a) mappings $Q: K \times K \rightarrow Y, g: K \rightarrow K, h: K \rightarrow K$, and $F: K \rightarrow Y$ are continuous;

(b) there exists a mapping $T: K \times K \rightarrow Y$ such that

(b1) $T(x, x) \geq 0, \quad \forall x \in K$;

(b2) $Q(x, g(y))-Q(x, h(x))+F(g(y))-F(h(x))-T(x, y) \geq 0$, $\forall x, y \in K$

(b3) the set $\{y \in K: T(x, y) \nsupseteq 0\}$ is convex for all $x \in K$; 
(c) there exists a nonempty compact convex subset $C$ of $K$ such that for all $x \in K \backslash C$ there exists $y \in C$ such that

$$
Q(x, g(y))-Q(x, h(x))+F(g(y))-F(h(x)) \nsupseteq 0 .
$$

Then (GMVF-IVIP) has a solution. Furthermore, the solution set of (GMVF-IVIP) is closed.

Proof. Define a set-valued mapping $G: K \rightarrow 2^{C}$ by

$G(y)=\{x \in C: Q(x, g(y))-Q(x, h(x))+F(g(y))-F(h(x)) \geq 0\}, \quad y \in K$.

For any $y \in K$, the assumption (a) implies that $G(y)$ is closed in $C$. Since every element $x \in \bigcap_{y \in K} G(y)$ is a solution of (GMVF-IVIP), we have to show that $\bigcap_{y \in K} G(y) \neq \emptyset$. Since $C$ is compact, it is sufficient to prove that the family $\{G(y)\}_{y \in K}$ has the finite intersection property. Let $\left\{y_{1}, y_{2}, \ldots, y_{n}\right\}$ be a finite subset of $K$ and set $B:=\overline{c o}\left(C \cup\left\{y_{1}, y_{2}, \ldots, y_{n}\right\}\right)$. Then $B$ is a compact and convex subset of $K$.

Define two set-valued mappings $F_{1}, F_{2}: B \rightarrow 2^{B}$ as follows:

$F_{1}(y)=\{x \in B: Q(x, g(y))-Q(x, h(x))+F(g(y))-F(h(x)) \geq 0\}, \quad y \in B$. and

$$
F_{2}(y)=\{x \in B: T(x, y) \geq 0\}, \quad y \in B .
$$

From the conditions (b1) and (b2), we have

$$
T(y, y) \geq 0
$$

and

$$
Q(y, g(y))-Q(y, h(y))+F(g(y))-F(h(y))-T(y, y) \geq 0 .
$$

Now Lemma 2.2 implies

$$
Q(y, g(y))-Q(y, h(y))+F(g(y))-F(h(y)) \geq 0
$$

and so $F_{1}(y)$ is nonempty. Similarly, we can prove that for any $y \in B, F_{1}(y)$ is closed. Since $F_{1}(y)$ is a closed subset of a compact set $B$, we know that $F_{1}(y)$ is compact. Next, we prove that $F_{2}$ is a KKM-mapping. Suppose that there exists a finite subset $\left\{u_{1}, u_{2}, \ldots, u_{n}\right\}$ of $B$ and $\lambda_{i} \geq 0(i=1,2, \ldots, n)$ with $\sum_{i=1}^{n} \lambda_{i}=1$ such that

$$
u=\sum_{i=1}^{n} \lambda_{i} u_{i} \notin \bigcup_{j=1}^{n} F_{2}\left(u_{j}\right) .
$$

Then

$$
T\left(u, u_{j}\right) \nsupseteq 0, \quad j=1,2, \ldots, n .
$$

From the condition (b3), we have

$$
T(u, u) \nsupseteq 0 .
$$


which contradicts the condition (b1). Hence $F_{2}$ is a KKM-mapping. On the other hand, from the condition (b2), we have

$$
F_{2}(y) \subset F_{1}(y), \quad \forall y \in B .
$$

In fact, $x \in F_{2}(y)$ implies that $T(x, y) \geq 0$ and by the condition (b2), we have

$$
Q(x, g(y))-Q(x, h(x))+F(g(y))-F(h(x))-T(x, y) \geq 0 .
$$

It follows from Lemma 2.2 that

$$
Q(x, g(y))-Q(x, h(x))+F(g(y))-F(h(x)) \geq 0
$$

and so $x \in F_{1}(y)$. Thus $F_{1}$ is also a KKM-mapping and Lemma 2.1 implies that there exists $x^{*} \in B$ such that $x^{*} \in F_{1}(y)$ for all $y \in B$. That is, there exists $x^{*} \in B$ such that

$$
Q\left(x^{*}, g(y)\right)-Q\left(x^{*}, h\left(x^{*}\right)\right)+F(g(y))-F\left(h\left(x^{*}\right)\right) \geq 0, \quad \forall y \in B .
$$

By assumption (c), we get $x^{*} \in C$. Moreover, $x^{*} \in G\left(y_{i}\right)$ for $i=1,2, \ldots, n$. Hence $\{G(y)\}_{y \in K}$ has the finite intersection property.

Since $Q, F, g$ and $h$ are continuous, the solution set of (GMVF-IVIP) is obviously closed.

Let $D=K$ in the condition (c) of Theorem 3.2. Then we have the following result.

Theorem 3.3. Let $K$ be a nonempty, compact and convex subset of $X$, and assume that the conditions $(a)$ and $(b)$ of Theorem 3.2 hold, then (GMVFIVIP) has a solution.

Proof. The conclusion follows directly from Theorem 3.2.

Theorem 3.4. Assume that $Q: K \times K \rightarrow Y$ is positively homogeneous in the second argument and continuous, $F: K \rightarrow Y$ is positively homogeneous and continuous, $g: K \rightarrow K$ is surjective and continuous, and $h: K \rightarrow K$ is continuous. If assumptions (b) and (c) in Theorem 3.2 hold, then (GMVF$I C P)$ has a solution. Furthermore, the solution set of $(G M V F-I C P)$ is closed.

Proof. The conclusion follows directly from Theorems 3.1 and 3.2. 
Example 3.1. Let $X=Y=R^{2}, K=P=R_{+}^{2}=[0, \infty) \times[0, \infty)$, and $C=[0,1] \times[0,1]$. Let

$$
g(x)=\left(\frac{x_{2}}{2}, \frac{x_{1}}{3}\right), \quad h(x)=\left(\frac{x_{2}}{3}, \frac{x_{1}}{4}\right), \quad F(x)=\left(x_{1}, 0\right)
$$

and $Q(x, y)=\left(y_{1}+y_{2}, 0\right)$ for any $x, y \in K$, with $x=\left(x_{1}, x_{2}\right)$ and $y=$ $\left(y_{1}, y_{2}\right)$. Then,

$$
Q(x, g(y))-Q(x, h(x))=\left(\left(\frac{y_{1}}{3}+\frac{y_{2}}{2}\right)-\left(\frac{x_{1}}{4}+\frac{x_{2}}{3}\right), 0\right)
$$

for any $x, y \in K$, with $x=\left(x_{1}, x_{2}\right)$ and $y=\left(y_{1}, y_{2}\right)$. If we set

$$
T(x, y)=\left(\left(\frac{y_{1}}{3}+y_{2}\right)-\left(\frac{x_{1}}{4}+\frac{2 x_{2}}{3}\right), 0\right)
$$

for any $x, y \in K$, with $x=\left(x_{1}, x_{2}\right)$ and $y=\left(y_{1}, y_{2}\right)$, then all assumptions in Theorem 3.2 hold. In fact, it is easy to see that $(0,0) \in K$ is a unique solution of (GMVF-IVIP).

Definition 3.1. Let $E \subset X, Q: E \times E \rightarrow Y, g: E \rightarrow E$ and $F: E \rightarrow Y$. We say that $Q$ and $F$ are locally non-positive at $x_{0} \in E$ with respect to $g$ if there exist a neighborhood $V\left(x_{0}\right)$ of $x_{0}$ and $z_{0} \in E \cap \operatorname{int} V\left(x_{0}\right)$ such that

$$
Q\left(x, g\left(z_{0}\right)\right)-Q(x, g(x))+F\left(g\left(z_{0}\right)\right)-F(g(x)) \leq 0, \quad \forall x \in E \cap \partial V\left(x_{0}\right),
$$

where $\partial V\left(x_{0}\right)$ denotes the boundary of $V\left(x_{0}\right)$.

Theorem 3.5. Let $K$ be a nonempty closed and convex subset of $X$. Assume that

(a) mappings $Q: K \times K \rightarrow Y, g: K \rightarrow K$ and $F: K \rightarrow Y$ are continuous;

(b) there exists a mapping $T: K \times K \rightarrow Y$ such that

(b1) $T(x, x) \geq 0, \quad \forall x \in K$;

(b2) $Q(x, g(y))-Q(x, g(x))+F(g(y))-F(g(x))-T(x, y) \geq 0$, $\forall x, y \in K$

(b3) the set $\{y \in K: T(x, y) \nsupseteq 0\}$ is convex for all $x \in K$;

(c) the mappings $Q$ and $F$ are locally non-positive at $x_{0} \in K$ with respect to $g$ and there exists a nonempty compact convex subset $C$ of $K \cap V\left(x_{0}\right)$ such that for all $x \in K \cap V\left(x_{0}\right) \backslash C$ there exists $y \in C$ such that

$$
Q(x, g(y))-Q(x, g(x))+F(g(y))-F(g(x)) \nsupseteq 0 ;
$$

(d) the set $\{y \in K: Q(x, g(y))-Q(x, g(x))+F(g(y))-F(g(x)) \geq 0\}$ is convex for all $x \in K$.

Then (GMVF-IVIP) has a solution in the neighborhood of $x_{0}$, that is, there exists $x^{*} \in K \cap V\left(x_{0}\right)$ such that

$$
Q\left(x^{*}, g(y)\right)-Q\left(x^{*}, g\left(x^{*}\right)\right)+F(g(y))-F\left(g\left(x^{*}\right)\right) \geq 0, \quad \forall y \in K .
$$


Proof. Since the mappings $Q$ and $F$ are locally non-positive at $x_{0} \in K$ with respect to the mapping $g$, without loss of generality, we can assume that $V\left(x_{0}\right)$ is closed and convex. Then, $K \cap V\left(x_{0}\right)$ is also closed and convex. From Theorem 3.2, (GMVF-IVIP) has a solution $x^{*} \in K \cap V\left(x_{0}\right)$ such that

$$
\begin{aligned}
Q\left(x^{*}, g(y)\right)-Q\left(x^{*}, g\left(x^{*}\right)\right)+F(g(y))-F\left(g\left(x^{*}\right)\right) & \geq 0, \\
& \forall y \in K \cap V\left(x_{0}\right) .
\end{aligned}
$$

Next we show

$$
Q\left(x^{*}, g(y)\right)-Q\left(x^{*}, g\left(x^{*}\right)\right)+F(g(y))-F\left(g\left(x^{*}\right)\right) \geq 0, \quad \forall y \in K .
$$

If $x^{*} \in K \cap \operatorname{int} V\left(x_{0}\right)$, then $V\left(x_{0}\right)-x^{*}$ is a neighborhood of the origin and so it is absorbing. For any $y \in K$, there exists $t \in(0,1)$ such that $t\left(y-x^{*}\right) \in V\left(x_{0}\right)-x^{*}$ and so $y_{t}=t y+(1-t) x^{*} \in K \cap V\left(x_{0}\right)$. It follows from (3.6) that

$$
Q\left(x^{*}, g\left(y_{t}\right)\right)-Q\left(x^{*}, g\left(x^{*}\right)\right)+F\left(g\left(y_{t}\right)\right)-F\left(g\left(x^{*}\right)\right) \geq 0 .
$$

From the condition (d), we have

$$
Q\left(x^{*}, g(y)\right)-Q\left(x^{*}, g\left(x^{*}\right)\right)+F(g(y))-F\left(g\left(x^{*}\right)\right) \geq 0 .
$$

Note $Q$ and $F$ are locally non-positive at $x_{0} \in E$ with respect to $g$. If $x^{*} \in K \cap \partial V\left(x_{0}\right)$, then there exists $z_{0} \in K \cap \operatorname{int} V\left(x_{0}\right)$ such that

$$
Q\left(x^{*}, g\left(z_{0}\right)\right)-Q\left(x^{*}, g\left(x^{*}\right)\right)+F\left(g\left(z_{0}\right)\right)-F\left(g\left(x^{*}\right)\right) \leq 0 .
$$

Similarly, for any $y \in K$, there exists a $t \in(0,1)$ such that $t\left(y-z_{0}\right) \in$ $V\left(x_{0}\right)-z_{0}$ and so $z_{t}=t y+(1-t) z_{0} \in K \cap V\left(x_{0}\right)$. It follows from (3.6) that

$$
Q\left(x^{*}, g\left(z_{t}\right)\right)-Q\left(x^{*}, g\left(x^{*}\right)\right)+F\left(g\left(z_{t}\right)\right)-F\left(g\left(x^{*}\right)\right) \geq 0 .
$$

Letting $t \rightarrow 0$ in (3.8), we obtain

$$
Q\left(x^{*}, g\left(z_{0}\right)\right)-Q\left(x^{*}, g\left(x^{*}\right)\right)+F\left(g\left(z_{0}\right)\right)-F\left(g\left(x^{*}\right)\right) \geq 0,
$$

which together with (3.7) implies that

$$
Q\left(x^{*}, g\left(z_{0}\right)\right)-Q\left(x^{*}, g\left(x^{*}\right)\right)+F\left(g\left(z_{0}\right)\right)-F\left(g\left(x^{*}\right)\right)=0 .
$$

From the condition (d), we have

$$
\begin{aligned}
& t Q\left(x^{*}, g(y)\right)+(1-t) Q\left(x^{*}, g\left(x^{*}\right)\right)-Q\left(x^{*}, g\left(z_{0}\right)\right)+t F(g(y)) \\
& +(1-t) F\left(g\left(x^{*}\right)\right)-F\left(g\left(z_{0}\right)\right) \geq 0 .
\end{aligned}
$$

Therefore,

$$
Q\left(x^{*}, g(y)\right)-Q\left(x^{*}, g\left(x^{*}\right)\right)+F(g(y))-F\left(g\left(x^{*}\right)\right) \geq 0 .
$$

This completes the proof.

Let $D=K$ in the condition (c) of Theorem 3.5. Then we have the following result. 
Theorem 3.6. Let $K$ be a nonempty, compact and convex subset of a real Banach space $X$, and assume that the condition $(a),(b)$ and $(d)$ of Theorem 3.5 and the following condition hold:

(c)' the mappings $Q$ and $F$ are locally non-positive at $x_{0} \in K$ with respect to the mapping $g$.

Then (GMVF-IVIP) has a solution in the neighborhood of $x_{0}$, that is, there exists $x^{*} \in K \cap V\left(x_{0}\right)$ such that

$$
Q\left(x^{*}, g(y)\right)-Q\left(x^{*}, g\left(x^{*}\right)\right)+F(g(y))-F\left(g\left(x^{*}\right)\right) \geq 0, \quad \forall y \in K .
$$

Theorem 3.7. Assume that

(a) $Q: K \times K \rightarrow Y$ is continuous and positively homogeneous in the second argument, $g: K \rightarrow K$ is continuous and surjective, and $F: K \rightarrow Y$ is continuous and positively homogeneous;

(b) there exists a mapping $T: K \times K \rightarrow Y$ such that

(b1) $T(x, x) \geq 0, \quad \forall x \in K$;

(b2) $Q(x, g(y))-Q(x, g(x))+F(g(y))-F(g(x))-T(x, y) \geq 0$, $\forall x, y \in K$

(b3) the set $\{y \in K: T(x, y) \nsupseteq 0\}$ is convex for all $x \in K$;

(c) the mappings $Q$ and $F$ are locally non-positive at $x_{0} \in K$ with respect to the mapping $g$, and there exists a nonempty compact convex subset $C$ of $K \cap V\left(x_{0}\right)$ such that for all $x \in K \cap V\left(x_{0}\right) \backslash C$ there exists $y \in C$ such that

$$
Q(x, g(y))-Q(x, g(x))+F(g(y))-F(g(x)) \nsupseteq 0 ;
$$

(d) the set $\{y \in K: Q(x, g(y))-Q(x, g(x))+F(g(y))-F(g(x)) \geq 0\}$ is convex for all $x \in K$.

Then (GMVF-IVCP) has a solution in the neighborhood of $x_{0}$, that is, there exists $x^{*} \in K \cap V\left(x_{0}\right)$ such that

$$
Q\left(x^{*}, g\left(x^{*}\right)\right)+F\left(g\left(x^{*}\right)\right)=0
$$

and

$$
\left.Q\left(x^{*}, g(y)\right)\right)+F(g(y)) \geq 0, \quad \forall y \in K \text {. }
$$

Proof. The conclusion follows directly from Theorems 3.1 and 3.5. 


\section{Stability RESUlts}

In this section, let $X$ be a real Banach space, $(Y, \leq)$ be an ordered Banach space induced by a pointed closed convex cone $P$, and $E$ be a nonempty convex subset of $X$. Let $\Lambda_{i}(i=1,2)$ be Hausdorff topological vector spaces (the parametric spaces), and let $K: \Lambda_{1} \rightarrow 2^{X}$ be a set-valued mapping such that for any $\lambda \in \Lambda_{1}, K(\lambda)$ is a nonempty closed and convex subset of $X$ with $K\left(\Lambda_{1}\right)=\bigcup_{\lambda \in \Lambda_{1}} K(\lambda) \subset E$. In this section, we establish some sufficient conditions for the upper semicontinuity and lower semicontinuity of the solution mapping of the general mixed vector $F$-implicit variational inequalities in Banach spaces.

Theorem 4.1. Assume that

(a) mappings $Q: \Lambda_{2} \times E \times E \rightarrow Y, g: E \rightarrow E, h: E \rightarrow E$, and $F: E \rightarrow Y$ are continuous;

(b) there exists a mapping $T: E \times E \rightarrow Y$ such that

(b1) $T(x, x) \geq 0, \quad \forall x \in E$;

(b2) $Q(\varepsilon, x, g(y))-Q(\varepsilon, x, h(x))+F(g(y))-F(h(x))-T(x, y) \geq 0$, $\forall x, y \in E, \varepsilon \in \Lambda_{2}$;

(b3) the set $\{y \in E: T(x, y) \nsupseteq 0\}$ is convex for all $x \in E$;

(c) $K: \Lambda_{1} \rightarrow 2^{X}$ is a continuous set-valued mapping such that for any $\lambda \in \Lambda_{1}, K(\lambda)$ is a nonempty, compact and convex subset of $X$.

Then, we have

(1) for every $(\lambda, \varepsilon) \in \Lambda_{1} \times \Lambda_{2}, S(\lambda, \varepsilon) \neq \emptyset$;

(2) the solution mapping $S: \Lambda_{1} \times \Lambda_{2} \rightarrow 2^{X}$ is upper semicontinuous on $\Lambda_{1} \times \Lambda_{2}$.

Proof. (1) For every $(\lambda, \varepsilon) \in \Lambda_{1} \times \Lambda_{2}, Q(\varepsilon, \cdot, \cdot), g, h, F$ and $K(\lambda)$ satisfy all conditions in Theorem 3.2. Then, from Theorem 3.2, $S(\lambda, \varepsilon) \neq \emptyset$.

(2) For every $(\lambda, \varepsilon) \in \Lambda_{1} \times \Lambda_{2}$,

$$
\begin{aligned}
S(\lambda, \varepsilon)=\left\{x^{*} \in K(\lambda): Q\left(\varepsilon, x^{*}, g(y)\right)-Q\left(\varepsilon, x^{*}, h\left(x^{*}\right)\right)+F(g(y))\right. \\
\\
\left.-F\left(h\left(x^{*}\right)\right) \geq 0, \forall y \in K(\lambda)\right\} .
\end{aligned}
$$

It follows from the condition (a) that $S(\lambda, \varepsilon)$ a closed subset of $K(\lambda)$ and so it is compact. We now prove that $S$ is upper semicontinuous. In fact, let a net $\left\{\left(\lambda_{\alpha}, \varepsilon_{\alpha}\right)\right\}$ be such that $\left(\lambda_{\alpha}, \varepsilon_{\alpha}\right) \rightarrow(\lambda, \varepsilon)$, and take a net $\left\{x_{\alpha}\right\}$ with $x_{\alpha} \in S\left(\lambda_{\alpha}, \varepsilon_{\alpha}\right)$. From Lemma 2.3, we only need to prove that there exist $x \in S(\lambda, \varepsilon)$ and a subnet $\left\{x_{\alpha_{i}}\right\}$ of $\left\{x_{\alpha}\right\}$ such that $x_{\alpha_{i}} \rightarrow x$. Since $x_{\alpha} \in K\left(\lambda_{\alpha}\right)$ and $K$ is upper semicontinuous, by Lemma 2.3, there exist $x \in K(\lambda)$ and a subnet $\left\{x_{\alpha_{i}}\right\}$ of $\left\{x_{\alpha}\right\}$ such that $x_{\alpha_{i}} \rightarrow x$. We now show 
that $x \in S(\lambda, \varepsilon)$. Suppose to the contrary that $x \notin S(\lambda, \varepsilon)$. Then, there exists $y \in K(\lambda)$ such that

$$
Q(\varepsilon, x, g(y))-Q(\varepsilon, x, h(x))+F(g(y))-F(h(x)) \nsupseteq 0 .
$$

Since $K$ is lower semicontinuous, by Lemma 2.3, for the above $y$, there exists a net $\left\{y_{n_{i}}\right\}$ such that $y_{n_{i}} \in K\left(\lambda_{n_{i}}\right)$ and $y_{n_{i}} \rightarrow y$. It follows from $x_{n_{i}} \in S\left(\lambda_{n_{i}}, \varepsilon_{n_{i}}\right)$ that

$Q\left(\varepsilon_{n_{i}}, x_{n_{i}}, g\left(y_{n_{i}}\right)\right)-Q\left(\varepsilon_{n_{i}}, x_{n_{i}}, h\left(x_{n_{i}}\right)\right)+F\left(g\left(y_{n_{i}}\right)\right)-F\left(h\left(x_{n_{i}}\right)\right) \geq 0$.

Since $Q, f, h$ and $F$ are continuous, from (4.2), we have

$$
Q(\varepsilon, x, g(y))-Q(\varepsilon, x, h(x))+F(g(y))-F(h(x)) \geq 0,
$$

which contradicts (4.1). Thus, $x \in S(\lambda, \varepsilon)$. This completes the proof.

Theorem 4.2. Assume that

(a) mappings $Q: \Lambda_{2} \times E \times E \rightarrow Y, g: E \rightarrow E$ and $F: E \rightarrow Y$ are continuous;

(b) there exists a mapping $T: E \times E \rightarrow Y$ such that

(b1) $T(x, x) \geq 0, \quad \forall x \in E$;

(b2) $Q(\varepsilon, x, g(y))-Q(\varepsilon, x, g(x))+F(g(y))-F(g(x))-T(x, y) \geq 0$, $\forall x, y \in E, \varepsilon \in \Lambda_{2}$;

(b3) the set $\{y \in E: T(x, y) \nsupseteq 0\}$ is convex for all $x \in E$;

(c) $K: \Lambda_{1} \rightarrow 2^{X}$ is a lower semicontinuous set-valued mapping such that for any $\lambda \in \Lambda_{1}, K(\lambda)$ is a nonempty, compact and convex subset of $X$;

(d) for any given $\left(\lambda_{0}, \varepsilon_{0}\right) \in \Lambda_{1} \times \Lambda_{2}$, there exist neighborhoods $U\left(\lambda_{0}\right)$ of $\lambda_{0}$ and $I\left(\varepsilon_{0}\right)$ of $\varepsilon_{0}$ such that for any $\lambda \in U\left(\lambda_{0}\right), \varepsilon \in I\left(\varepsilon_{0}\right)$ and $x \in K(\lambda)$, the mappings $Q(\varepsilon, \cdot, \cdot)$ and $F$ are locally non-positive at $x$ with respect to the mapping $g$;

(e) the set $\{y \in E: Q(\varepsilon, x, g(y))-Q(\varepsilon, x, g(x))+F(g(y))-F(g(x)) \geq 0\}$ is convex for all $x \in E, \varepsilon \in \Lambda_{2}$.

Then, we have

(1) for every $(\lambda, \varepsilon) \in \Lambda_{1} \times \Lambda_{2}, S(\lambda, \varepsilon) \neq \emptyset$;

(2) the solution mapping $S: \Lambda_{1} \times \Lambda_{2} \rightarrow 2^{X}$ is lower semicontinuous at $\left(\lambda_{0}, \varepsilon_{0}\right)$.

Proof. (1) For every $(\lambda, \varepsilon) \in \Lambda_{1} \times \Lambda_{2}, Q(\varepsilon, \cdot, \cdot), g, F$ and $K(\lambda)$ satisfy all conditions in Theorem 3.6. Then, from Theorem 3.6, $S(\lambda, \varepsilon) \neq \emptyset$.

(2) For every $(\lambda, \varepsilon) \in \Lambda_{1} \times \Lambda_{2}$,

$$
\begin{aligned}
& S(\lambda, \varepsilon)=\left\{x^{*} \in K(\lambda): Q\left(\varepsilon, x^{*}, g(y)\right)-Q\left(\varepsilon, x^{*}, g\left(x^{*}\right)\right)+F(g(y))\right. \\
&\left.-F\left(g\left(x^{*}\right)\right) \geq 0, \forall y \in K(\lambda)\right\} .
\end{aligned}
$$


Let a net $\left\{\left(\lambda_{\alpha}, \varepsilon_{\alpha}\right)\right\} \subset \Lambda_{1} \times \Lambda_{2}$ be such that $\left(\lambda_{\alpha}, \varepsilon_{\alpha}\right) \rightarrow\left(\lambda_{0}, \varepsilon_{0}\right)$. For any $x_{0} \in S\left(\lambda_{0}, \varepsilon_{0}\right)$, by Lemma 2.3 (ii), it is sufficient to prove that there exists a net $\left\{x_{\alpha}\right\}$ with $x_{\alpha} \in S\left(\lambda_{\alpha}, \varepsilon_{\alpha}\right)$ such that $x_{\alpha} \rightarrow x_{0}$.

In fact, since $x_{0} \in K\left(\lambda_{0}\right)$ and $K$ is lower semicontinuous, by definition, for any neighborhood $V\left(x_{0}\right)$ of $x_{0}$, there is a neighborhood $H$ of $\lambda_{0}$ with $H \subset U\left(\lambda_{0}\right)$ such that for any $\lambda \in H, K(\lambda) \cap V\left(x_{0}\right) \neq \emptyset$. Since $\lambda_{\alpha} \rightarrow \lambda_{0}$ and $\varepsilon_{\alpha} \rightarrow \varepsilon_{0}$, there exist a neighborhood $B$ of $\varepsilon_{0}$ with $B \subset I\left(\varepsilon_{0}\right)$ and some $\alpha_{0}$ such that for any $\alpha \geq \alpha_{0}, \lambda_{\alpha} \in H$ and $\varepsilon_{\alpha} \in B$. Thus, $K\left(\lambda_{\alpha}\right) \cap V\left(x_{0}\right) \neq \emptyset$. By the condition (d) and Theorem 3.6, for any $\alpha \geq \alpha_{0}$, there exists $x_{\alpha} \in$ $K\left(\lambda_{\alpha}\right) \cap V\left(x_{0}\right)$ such that

$$
Q\left(\varepsilon_{\alpha}, x_{\alpha}, g(y)\right)-Q\left(\varepsilon_{\alpha}, x_{\alpha}, h\left(x_{\alpha}\right)\right)+F(g(y))-F\left(h\left(x_{\alpha}\right)\right) \geq 0, \quad \forall y \in K\left(\lambda_{\alpha}\right) .
$$

That is, $x_{\alpha} \in S\left(\lambda_{\alpha}, \varepsilon_{\alpha}\right)$. Since $V\left(x_{0}\right)$ is arbitrary and $x_{\alpha} \in V\left(x_{0}\right)$ for all $\alpha \geq \alpha_{0}$, we have $x_{\alpha} \rightarrow x_{0}$. The proof is completed.

From Theorems 4.1 and 4.2, we can derive the continuity of the solution mapping.

Theorem 4.3. Assume that

(a) mappings $Q: \Lambda_{2} \times E \times E \rightarrow Y, g: E \rightarrow E$ and $F: E \rightarrow Y$ are continuous;

(b) there exists a mapping $T: E \times E \rightarrow Y$ such that

(b1) $T(x, x) \geq 0, \quad \forall x \in E$;

(b2) $Q(\varepsilon, x, g(y))-Q(\varepsilon, x, g(x))+F(g(y))-F(g(x))-T(x, y) \geq 0$, $\forall x, y \in E, \varepsilon \in \Lambda_{2}$

(b3) the set $\{y \in E: T(x, y) \nsupseteq 0\}$ is convex for all $x \in E$;

(c) $K: \Lambda_{1} \rightarrow 2^{X}$ is a continuous set-valued mapping such that for any $\lambda \in \Lambda_{1}, K(\lambda)$ is a nonempty, compact and convex subset of $X$;

(d) for any $\left(\lambda_{0}, \varepsilon_{0}\right) \in \Lambda_{1} \times \Lambda_{2}$, there exist neighborhoods $U\left(\lambda_{0}\right)$ of $\lambda_{0}$ and $I\left(\varepsilon_{0}\right)$ of $\varepsilon_{0}$ such that for any $\lambda \in U\left(\lambda_{0}\right), \varepsilon \in I\left(\varepsilon_{0}\right)$ and $x \in K(\lambda)$, the mappings $Q(\varepsilon, \cdot, \cdot)$ and $F$ are locally non-positive at $x$ with respect to the mapping $g$;

(e) the set $\{y \in E: Q(\varepsilon, x, g(y))-Q(\varepsilon, x, g(x))+F(g(y))-F(g(x)) \geq 0\}$ is convex for all $x \in E, \varepsilon \in \Lambda_{2}$.

Then, we have

(1) for every $(\lambda, \varepsilon) \in \Lambda_{1} \times \Lambda_{2}, S(\lambda, \varepsilon) \neq \emptyset$;

(2) the solution mapping $S: \Lambda_{1} \times \Lambda_{2} \rightarrow 2^{X}$ is continuous on $\Lambda_{1} \times \Lambda_{2}$.

Proof. (1) For every $(\lambda, \varepsilon) \in \Lambda_{1} \times \Lambda_{2}, Q(\varepsilon, \cdot, \cdot), g, F$ and $K(\lambda)$ satisfy all conditions in Theorem 3.6. From Theorem 3.6, we know that $S(\lambda, \varepsilon) \neq \emptyset$.

(2) The conclusion follows from Theorems 4.1 and 4.2. The proof is completed. 
Acknowledgements. The authors are grateful to the referees for their valuable comments and suggestions.

\section{References}

[1] Anh, L. Q., Khanh, P. Q., Semicontinuity of the solution set of parametric multivalued vector quasiequilibrium problems, J. Math. Anal. Appl. 294 (2004), 699-711.

[2] Carbone, A., A note on complementarity problem, Internat. J. Math. Math. Sci. 21(3) (1998), 621-623.

[3] Chen, G. Y., Yang, X. Q., The vector complementary problem and its equivalences with vector minimal element in ordered sppaces, J. Math. Anal. Appl. 153 (1990), $136-158$.

[4] Chen, G. Y., Huang, X. X., Yang, X. Q., Vector Optimization: Set-Valued and Variational Analysis, Springer-Verlag, Berlin-Heidelberg, 2005.

[5] Cottle, R. W., Yao, J. C., Pseudomonotone complementarity problems in Hilbert spaces, J. Optim. Theory Appl. 78 (1992), 281-295.

[6] Cottle, R. W., Pang, J. S., Stone, R. E., The Linear Complementarity Problem, Academic Press, New York, 1992.

[7] Facchinei, F., Pang, J. S., Finite-dimensional Variational Inequalities and Complementarity Problems, Springer-Verlag, New York, 2003.

[8] Fan, K., A generalization of Tychonoff's fixed point theorem, Math. Ann. 142 (1961), 305-310.

[9] Fang, Y. P., Huang, N. J., The vector F-complementary problems with demipseudomonotone mappings in Banach spaces, Appl. Math. Lett. 16 (2003), 1019-1024.

[10] Ferro, F., A minimax theorem for vector-valued functions, J. Optim. Theory Appl. 60 (1989), 19-31.

[11] Giannessi, F., Theorems of alterative, quadratic programs and complementarity problems, in "Vatiutional Inequalities and Complementarity Problems", R. W. Cottle, F. Giannessi and J. L. Lions (eds.), Wiley, New York, 1980.

[12] Giannessi, F., Mastroeni, G., Pellegrini, L., On the theory of vector optimization and variational inequalities. Image space analysis and separation, in "Vector Variational Inequalities and Vector Equilibria", Nonconvex Optim. Appl. 38, Kluwer Acad. Publ., Dordrecht, 2000, 153-215.

[13] Huang, N. J., Li, J., F-Implicit complementarity problems in Banach spaces, Z. Anal. Anwendungen 23 (2004), 293-302.

[14] Huang, N. J., Li, J., Thompson, H. B., Stability for parametric implicit vector equilibrium problems, Math. Comput. Modelling 43 (2006), 1267-1274.

[15] Isac, G., Complementarity Problems, Lecture Notes in Math. 1528, Springer-Verlag, New York, 1992.

[16] Isac, G., Topological Methods in Complementarity Theory, Kluwer Acad. Publ., Dordrecht-Boston-London, 2000.

[17] Lee, B. S., Khan, M. F., Salahuddin, Vector F-implicit complementarity problems with corresponding variational inequality problems, Appl. Math. Lett. 20 (2007), 433-438.

[18] Li, J., Huang, N. J., Vector F-implicit complementarity problems in Banach spaces, Appl. Math. Lett. 19 (2006), 464-471.

[19] Yang, X. Q., Vector complementarity and minimal element problems, J. Optim. Theory Appl. 77 (1993), 483-495. 
[20] Yin, H. Y., Xu, C. X., Zhang, Z. X., The complementarity problems and its equivalence with the least element problem, Acta Math. Sinica 44 (2001), 679-686.

KE-QING WU

DePARTMENT OF MATHEMATiCs

JIANGXI UNIVERSITY

of SCIENCE AND TECHNOLOGY

Ganzhou, Jiangxi, P. R. China

E-MAIL: WUKEQING622@SINA.COM
NAN-JING HUANG

DEPARTMENT OF MATHEMATiCs

SiCHUAN UNIVERSITY

Chengdu, Sichuan 610064

P. R. ChinA

E-MAIL: NANJINGHUANG@HOTMAIL.COM 